\title{
Digital Transformation and Ecosystem of Smart Pillows
}

\author{
Xingyu Liu ${ }^{1}$, Shijia Yin ${ }^{2}$, Yi Li ${ }^{3}$, Fei Wu ${ }^{4}$, Ziyue $\mathrm{Zhu}^{5}$ \\ ${ }^{1}$ Staten Island Academy, Staten Island, New York, 10304, United States \\ ${ }^{2}$ Shenzhen College of International Education, Shenzhen, Guangdong 518040, China \\ ${ }^{3}$ Vanke Meisha Academic School, Shenzhen, Guangdong 518085, China \\ ${ }^{4}$ Nansha College Preparatory Academy, Guangzhou, Guangdong 511458, China \\ ${ }^{5}$ Yangzhou Middle School of Jiangsu Province, Yangzhou, Jiangsu 225000, China \\ *s16113.yin@stu.scie.com.cn
}

\begin{abstract}
This paper discusses how smart pillows relate to and benefit from digital platforms and ecosystems. With increasing demand for products that improve quality of life, many firms are focusing on products that improve the quality of our sleep. Modern digital technologies make it possible to expand the functionalities of sleep products and offer new smart features that can help people sleep better. These new digital features are possible because of two advances driven by modern digital technologies. The first is about tapping new kinds of data through technologies like sensors. And the second concerns digital ecosystems, or networks of data generators and recipients possible because of the exponential rise of the Internet of Things (IoT). This paper highlights how these advances apply to the specific case of smart pillows.
\end{abstract}

Keywords: Data, Digital Platform, Production Ecosystem, Consumption Ecosystem

\section{INTRODUCTION}

We clearly live in a digital world today. The most successful enterprises in this era are digital companies such as, Tencent, Alibaba, Google and Uber. Traditional companies, that do not adapt to this new digital world are finding themselves becoming irrelevant. Hui Yuan, a famous juice producing company, for example, went bankrupt as they failed to adapt in time. Eastman Kodak, the most famous camera company before, went bankrupt because it insisted on producing films and didn't keep up with the trend of digital cameras in time. Not surprisingly many traditional firms have started to consider adopting digital technologies and making significant changes to their prevailing business models. They are looking at new ways of unlocking the value of data in their enterprises.

One such industry undergoing digital transformation is the sleep products industry. Many firms in this industry have adopted sensors to expand the features of traditional pillows. For example, Huawei launched a smart pillow last year, which won a lot of praises for its medium price, comfortable materials, and high-tech functions. Xiaomi, as competing companies to Huawei, also published a smart pillow that mainly focuses on music playing function. Although both products have achieved good results, there are still many functions that we can improve on. In other words, there is much that firms can do to unlock the potential of smart pillows.

In this paper, we demonstrate some ideas about how we can improve on existing smart pillow industries and popularize smart pillows to the general publics from the aspects of both production and consumption ecosystems. As a rising industry, there are currently more than 60 thousand companies related to sleep and more than a half of them were founded after 2015. This number is growing by about 300 annually. The current value of sleep industry is about 400 billion, which expected to be 1 trillion in 2030. These statistics provide hard evidence of the massive impact digital technologies can have on the sleep industry.

The paper is structured as follows. We start with a literature review, and then there is a picture in the framework which roughly explains the product structure. Next, we discuss how production ecosystem and consumption ecosystem work on smart pillows, respectively. Finally, we analyze the market and discuss some strategies. 


\section{LITERATURE REVIEW}

\subsection{New data of smart pillows}

The big transformation of data has come about with the rise in sensors. Sensors market it possible for products to generate interactive data. Interactive data has a realtime element which over time can be stored and archived. But the two have different properties ${ }^{[1]}$. Real-time data provides real-time deep insights and easier sharing. Here is a good example: sensors in the smart pillows can collect data in real time which means that the data will be uploaded to the system as individuals use the pillows. Real-time data is starting to be combined with archival data - it is no longer just being kept in a cluttered format. It is being organized into a personal profile, which make the data customized. Sensors in the smart pillows, for instance, provide more users' data to the legacy company, and thus the company can research collected data to develop specific functions of pillows to satisfy each customer's preferences. In short, it is a snowball. Even if the number of data is too small for being significant for a company at the beginning, as the number of users increases, more and more data will be obtained, making the company's database not only massive but also powerful.

To leverage the value of data, a firm needs to build digital ecosystems. Digital ecosystems are networks of data generators and recipients. The newly introduced system contains three parts: sensors, transmission interface and services. Community decides to use interactive data which allows the software programs to interact with each other. The reason why the API is necessary is that it becomes an integral part of the process which continuously collects and combines data between producers and consumers. For example, when consumers use the application of smart pillows, they are redirected to a page offering suggestions through an app that provides data.

\subsection{Digital Ecosystems of Legacy Firms}

Digital ecosystems are driven by new digital technologies that change the nature and scope of traditional interdependencies. These interdependencies translate into digital networks of data generators and data recipients. For instance, Didi company transformed from traditional business of taxi, generate value between the driver and customers through the digital ecosystem. The scopes and significance of digital ecosystems today extend far beyond traditional value chains and traditional industrial structures. In new digital ecosystems, the scope of traditional products, services, and their potential value chains is expanding as never before, and new opportunities to deliver value are growing ${ }^{[2]}$. To understand this enlarged scope it is useful to see digital ecosystems as a combination of production and consumption ecosystems.

\subsubsection{Production Ecosystems:}

The production ecosystem reinforces and extends the traditional value of the products and service and it consists of interdependencies enmeshed in a value chain, such as producing and selling a product or delivering a service to a customer. It is thus a network of data generators and recipients involved in the producing and selling of a product.

\subsubsection{Consumption Ecosystems:}

The consumption ecosystem consists of interdependencies including after a product is sold and is consumed. Also, the consumption ecosystem personifies what is new about interdependencies, which allows for more plug-and-play options. Equipped with sensors and Internet of Things (IoT) connectivity with a variety of other objects, the traditional products are digitized and generate new features to meet users' needs. Such digitally driven ecosystems are consumer ecosystems because of the interdependencies between different objects or third-party entities that complement the data generated when products are used or consumed ${ }^{[2]}$. These new interdependencies based on complementarity are different from value chain interdependencies ${ }^{[3,4]}$. Thus, unlike production ecosystems that enrich traditional value chain interdependencies, consumer ecosystems are the result of the proliferation of a different set of interdependencies outside the value chain ${ }^{[1]}$. Based on above theories, how smart pillows can expand its competitive scope by using new kinds of data and building new digital ecosystems will be offered.

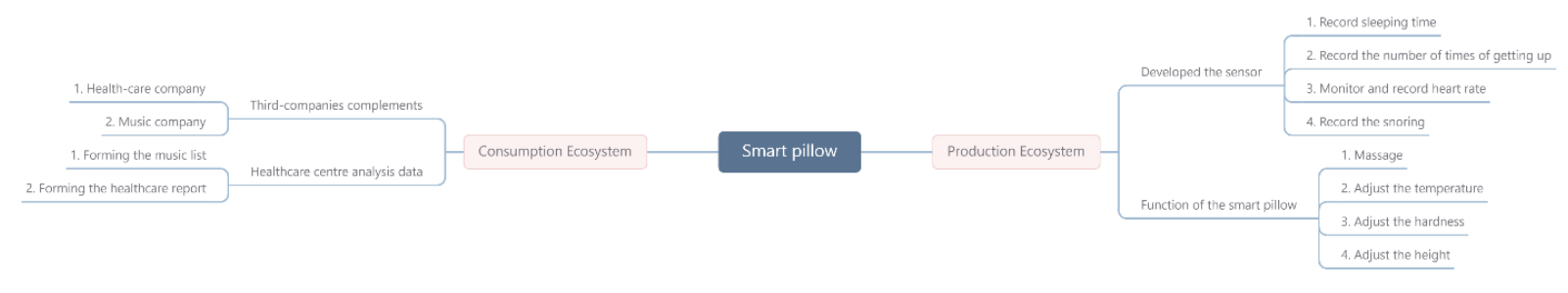

Figure 1 The framework of smart pillow 


\section{FRAMEWORK}

\subsection{Value Generated within Production ecosystems}

Production ecosystems arise from digitally connected sensing units embedded in an amalgamation of interdependent entities that contribute to a firm's value proposition through its value chain ${ }^{[2]}$. The smart pillow, a new generation pillow added sensors and modified from the traditional pillow construction that includes pillowcase and filling, can collect users' data and further form a production ecosystem. Within the production ecosystem of smart pillows, the value of product-in-use information through exchanges will be intensified ${ }^{[5]}$, with outcomes that largely reinforce the sleep quality and satisfy customers' higher requirements. For instance, the smart pillow will intelligently change the height and softness according to the users' data of head and neck position towards the pillow.

Here are two ways to create value or increase revenue. The first one is to utilize the mass-customization. Through customizing product features and services that can be micro-targeted to specific users, which would not have been possible without the sensors ${ }^{[2]}$. Our smart pillows have a variety of functions to meet the diverse needs of people of different ages.

\subsection{Function of Mass-Customization}

To collect data information of each user, the sensor is included in the smart pillows. The first function of the sensor is our products can record sleep time in order to reflect people's sleep and health. It's difficult for users to pay attention to their sleep problems because most people don't have any data to show their problems. The second is that the smart pillows can record the number of times to get up. The record of the number of times to get up shows the quality of their sleep quality. The third function is that our smart pillows can monitor and record heart rate. Detecting and recording heart rate can help users such as elderly patients with hypertension. When their heart rate or blood pressure is too high or too low, the pillow will send a signal in time so that the patient can be treated in time. Thus, they can have a safer sleep environment. The last function is that it can record the snoring. Snoring is generally caused by narrow respiratory tract, which will cause daytime sleepiness, headache, decreased working ability, and other problems. Our smart pillows can automatically record the frequency and sound of snoring so that customers can find and solve problems in time.

Based on the work of this sensor, the smart pillow also has a variety of functions. The first function is our product can help relax and protect people's cervical spines through an inside massage machine and adjustment of curvature so it may relax the head and cervical spine for people. According to the international authoritative medical institutions, more than $95 \%$ of the 100 sudden death cases are complications caused by cervical spondylosis. At the same time, cervical spondylosis also induces hypertension, diabetes, cardiovascular and cerebrovascular diseases, gastrointestinal diseases, and even cancer. Cervical spondylosis is becoming younger and younger; Eventually, the common diseases of the elderly are becoming younger and younger. Our pillow is to protect people's cervical spine and relieve people's cervical spine pressure through massaging. It is not uncommon that since young people in today's society work harder than before, their cervical spine health problems are becoming more and more serious. Poor cervical spine will cause dizziness, headache, and memory loss, which will greatly reduce people's quality of life and work efficiency. By using our products, although it is not guaranteed that all illnesses can be completely cured, some health issues can be effectively alleviated. Sensor can be used to inform people of cervical health concerns through mobile phones in time, and to predict the customer's potential for related diseases in advance.

The second function is that these smart pillows can adjust the temperature, in a more specific way, heat the smart pillows in winter and cool them in summer to have a better and more comfortable sleeping environment. Temperature is a major factor in sleep quality. ${ }^{[6]}$ Comfortable pillow temperatures can make people fall asleep faster and more relaxed. Sensor can also record the temperature of the user's head in time, in order to let the pillows to adjust to a suitable temperature. The next function of our products is that they can adjust the hardness. Our smart pillows can automatically adjust the soft and hard to meet people's diverse preferences and sleep needs since a part of people prefer harder pillows while others prefer softer pillows. This function can greatly improve the user's sleep quality. By recording sleep data, sensors can determine whether a client slept better with a hard pillow or a soft one.

The last function is height adjustment. The automatic height adjustment can change the height of the pillows and find a more comfortable position for users, for example, younger customers or female customers can turn their pillows down, and older customers or male customers can turn their pillows up. Customers can easily adjust the pillow to the height they like. The height of the headpiece can also be recorded by sensor, which can show the quality of sleep and so on the phone, allowing the customer to know their needs and health status. These 
types of specific services can generate new revenue streams.

\subsection{Function of Predictive Maintenance}

Furthermore, the second opportunity for value creation comes from predictive maintenance. Because sensors can monitor each component as it is used, they can sense and alert to failures before they occur. Predictive maintenance services are exceptionally valuable when the cost of unexpected failures is nontrivia ${ }^{[2]}$. As mentioned earlier, there are several sensors in the smart pillow. Besides the functions mentioned before, the sensors have another crucial feature: detecting possible breakdowns. For instance, typically, the massager in a smart pillow needs to be repaired due to extended use. When a certain length of use is reached, the sensor will light up or make an audible sound to alert the user when the function is used again. In the event that the user opens the massage function for a long time, such as forgetting to close, the smart pillow will automatically close the massage function as a way to increase the service life of the massager. Therefore, before the entire massager is destroyed, the user can advance maintenance, thus saving the cost of replacing the massager or even repurchase a new smart pillow. In this way, our smart pillow can attract more users.

Lastly, according to the Analysis on the acceptance of complaints from China Consumers Association (CSA) on 29 th July, there are more than $30 \%$ of the complaints were about after-sales service, an increase of $4 \%$ compared with last year. Moreover, the president of CSA pointed out "All products should be consumer-centered. Firms should pay attention to consumers' feedbacks and effectively improve after-sales services and customer complaints." Based on these statistics and suggestions, the after-sales service of sleep pillows should be improved. As part of the smart pillow industry, product maintenance is very important, so we include free consultation and maintenance services in the after-sales service of this smart pillow. The specific scheme is that as long as the prime membership is valid, customers can enjoy whole-life free maintenances for smart pillows.

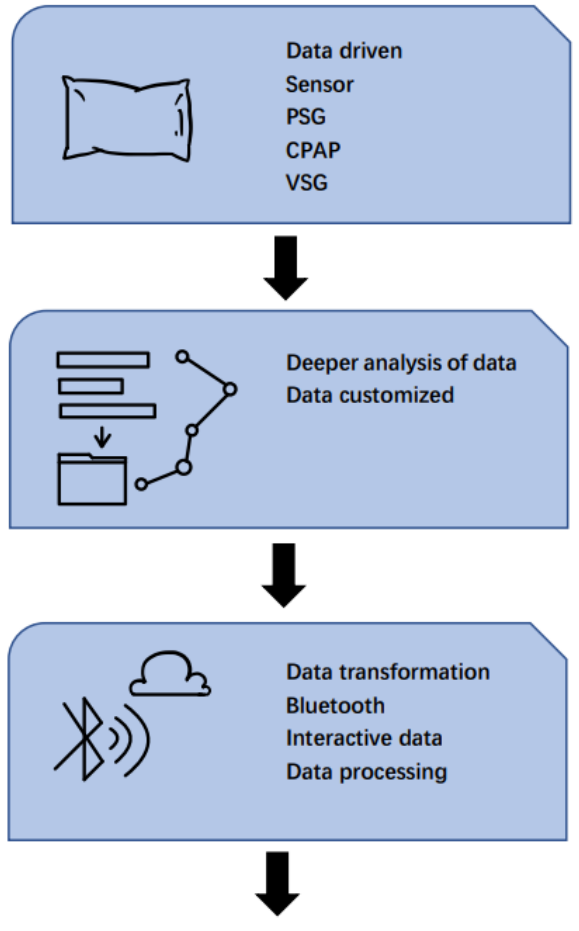

Applications

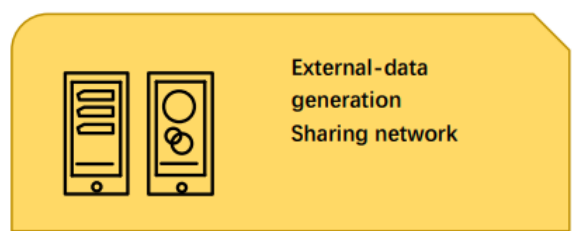

Figure 2 The process from sensor to the application platform.

\subsection{Value Generated within Consumption ecosystems}

To succeed in today's competitive marketplace, companies need to be customer-centered. They must win customers from competitors, then keep and grow them by delivering greater value. The physical product, in this case a pillow, is the main or core product. The services and accessories that surrounding it is called the augmented product, which support the core product. Most of the pillow products that exist on the market today include only interdependencies existing on the production side. However, we are going to move into a whole new area --- providing advice services and music services --- which is known as the consumption ecosystem.

\subsubsection{Cooperation with Healthcare Company}

Given that our pillows already have some datadriven, we choose to cooperate with third-party organizations for data sharing, such as music companies and healthcare companies. For instance, a healthcare center called ECMOHO is chosen as one of the thirdcomplement companies. It is established in 2011 and 
headquartered in Shanghai with three branches, which is Hong Kong, Japan, and Korea. As a comprehensive solution provider for China's non-medical and health market, focusing on omni-channel retail services, global marketing, customer service and global warehouse supply chain services, and has a rich service industry chain, naturally, it becomes a reliable partner. In 2014, the company has established cross-border warehouses and started cross-border business. On the client side of the new mobile application jointly launched by the three companies, customers can enjoy two basic services, including personal health reports from the healthcare company and exclusive music lists from the music company. The healthcare company can also provide customers with the service of "online doctor": they can make health consultations online in real-time and provide treatment recommendations for some diseases. As our company collects more customer data and shares it with the healthcare company, they can conduct further research on disease prevention and treatment options, considering the digital healthcare will bring benefit in the aspect of, self-harm prevention and risk \& disease management. To the customers, they will also receive more relevant and effective health analysis reports. Also, we can further modify and upgrade the performance and function of the product according to the customer's response and achieve low-cost innovative transformation.

After the sensor inside the pillow collects enough data, it will send the data to the cloud or the database for the healthcare company to fetch the data to integrate and analyze a complete set of personal healthcare reports, and the whole set of the report will be sent back to the database then transmit to the app that our company established. In the healthcare report, users are given their sleep status at the time of sleep, including heart rate, blood oxygen, and deep sleep time. With the help of a Bluetooth-enabled smartphone, users can access the analyzed data, providing data support for understanding and targeted improvement of sleep quality. Through the cloud server, doctors and relatives can remotely view the user's sleep data and timely understand the user's health condition and effective advices will be provided by the doctors. For example, when people spend a long time between going to bed and falling asleep, which means they have insomnia, doctors can offer physical and psychological help. In the case of insomnia, doctors may give biological advice --- drink a hot glass of milk before bed, soak your feet in hot water, don't wat too much, avoid strenuous exercise. Or don't think about anything, adjust your mind and relax which are psychological advices. If customers have a need for real-time online consultation, they can also consult with expert doctors provided by the healthcare company on the app.

\subsubsection{Cooperation with Music Company}

Music does influence sleep quality. As a beneficial way to improve sleep quality ${ }^{[7]}$, we decided to add a music playing function in the smart pillow by cooperating with other companies. We create an exclusive music list for each customer through the data analysis provided by the healthcare company and the music company. The music list is personalized for individual customers for scenarios such as sleep and morning calls ${ }^{[8]}$. For example, for customers who wake up suddenly and experience dizziness or discomfort, the song list will play soothing and gentle music 10 minutes before the morning alarm. There will be some negative effects, however, to listen to music continuously during sleep. Research from

《Phycological Science》 written by Dr. Micheal Scullin mentions "people who experienced the "earworm effect", that is, the phenomenon that people often repeat the song or melody in their mind when a song or melody is played repeatedly, woke up more at night, spent more time in light sleep, and were more difficult to fall asleep". How to ensure that music does not affect sleep quality has become a pivotal question. To avoid the negative effects of sleep quality brought by the music playing functions of smart pillows, there will be a timing function in the mobile application that allows customers to time their music. If there are no customer settings, the music will automatically stop after playing for 30 minutes.

\section{MARKETING PLAN}

\subsection{Existed market}

As a rising industry, there are currently more than 60 thousand company that are related to sleep and more than a half of them were founded after 2015. The current value of sleep industry is about 400 billion, which expected to be 1 trillion in 2030. Smart Pillows Market 2021 is estimated to clock a modest Compound Annual Growth Rate (CAGR) of $46.1 \%$ during the forecast period 2021 2026 With Top Countries Data. Global Smart Pillows market is valued at 74 million USD in 2020 and is expected to reach 1076 million USD by the end of 2026, growing at a CAGR of 46.1\% during 2021-2026 [9]. Therefore, we put more value on investing the smart pillows because the prospect of smart pillows is very promising. 
Table 1. Potential Rivals from both China and US

\begin{tabular}{|l|l|l|l|l|}
\hline Brands & Prices & Special functions & Materials & Smart? \\
\hline Lofty & $49.99 \sim 69.99$ USD & $/$ & Memory foam & No \\
\hline Cozy Earth & 279 USD with 20\% off & Temperature regulating & Mulberry silk & Yes \\
\hline HUAWEI & 449 RMB & Detections* & Latex & Yes \\
\hline Sleepwhale & 999 RMB & Adjustable height & Memory foam & Yes \\
\hline
\end{tabular}

*Table 1 shows the functions, prices, and other information of several quite popular and highly accepted smart pillows in the market.

*Detections include heart rates, breathe rates, flipping frequencies, periods of sleep,

As mentioned in Table 1, some firms have launched smart pillows with different functions. Through investigations and costumers' responses, we discover some problems that can be improved, for example from costumers' responses about Huawei smart pillows, people gradually find that the data Huawei detects are not useful, such as how many times you turn over when you sleep and how many times you leave bed. By doing surveys, we found that the reason why Huawei's data is not useful is because the company they cooperate with is an old household textile enterprise. Neither company has health-related experiences.

\subsection{Competitive Strategies}

Through the survey, we found that the reason why smart pillows cannot be popularized is that people prefer sleep drugs and smart mattresses than pillows. There are three main reasons for this phenomenon, insufficient knowledge about smart pillows, people's hesitations about new products, and the most important, not enough costumers. To solve these problems, we discuss and decide on the following plans.

\subsubsection{Focusing on self-media platforms.}

With the growing popularity of self-media platforms, such as TikTok and Instagram, in recent years, online advertising and sales has become an indispensable part for a new product. Such network trend actually has a positive effect to our pillows since it can help us let the public know our products in the shortest time and help us better integrate into the current market. We will study and consult about advertising strategies from companies that get huge popularities through short videos and then operate successfully, for example Genki forest, a soda company that increases its market value by 3 times in only one year. We believe that through short videos, we can make the public gain the relevant knowledge and experience of smart pillows quickly.

\subsubsection{Cooperating with experienced and branded health institutions.}

Indeed, it is difficult for us to compete with companies that already have a customer base. To catch up their progress, we decide to cooperate with a listed health organization called ECMOHO. Through working with them, we share our user data in exchange for their supports, including sharing their costumer group with us and providing our users the most customized, specific and real-time advice in order to ensure our customers' health. Also, we find out that the company we are going to work with is in short of user data. From this cooperation, we can not only improve ourselves, but also improve our partners by providing the data they need.

\subsection{Pricing}

Primarily, we set the smart pillow's price at $899 \mathrm{RMB}$ in China and 159 USD for other countries (if available in the future). Compared with the existing products in the market, the price is above medium. As sales increase, we will offer some special discounts to customers occasionally. For people who are willing to pay for better services, we introduce the Prime Membership that costs 399 RMB annually, which is only about 1.1 RMB per day. Membership services include real-time expert advice, simple pillow adjustment (including temperature, height, and music), and sleep data detections. There will also be special bundles containing both pillows and memberships for only 888 RMB. This bundle is strongly suggested for its conveniences and cheaper prices. (Only applies to the first payment by a new customer). To achieve the goal of better network effects, we also provide free opportunities for people who are not willing to purchase the prime membership. They will be able to experience part of the functions, such as detection function, in the app by sharing our app and suggesting one new costumer for us. 


\section{CONCLUSION}

With the development of the Internet and IoT technology, the whole smart furniture market will grow at a high speed. As an important part of smart furniture, smart pillows will be popularized in the future and bring great benefits to society. By adding sensors and cooperating with external companies, the function of pillows can be further improved. As a new industry, there must be some concerns and concerns, for example, some customers who have never used similar products may not be able to accept such relatively high prices. We believe that with the popularity of smart pillows and the increase of people's future income, these problems will be solved.

Also, there are some technology difficulties. Accessing internal databases is usually faster and cheaper, but they have some problems. Because internal information is often collected for other purposes, it can be incomplete or incorrectly documented for marketing decisions. In this era, data will also age rapidly, because it is constantly updated, which is the old data becomes worthless. Keeping the database up to date takes a lot of effort, which is not only a human barrier, but also greatly difficult to manage. Finally, managing and mining the vast amounts of information produced by large companies requires very complex equipment and technology, which is also a big cost [10].

\section{REFERENCES}

[1] Praveen K. Kopalle \& Kumar V. \& Subramaniam M. (2020) "How legacy firms can embrace the digital ecosystem via digital customer orientation," Journal of the Academy of Marketing Science, Springer, vol. 48(1), pages 114-131, January.

[2] Subramaniam M. \& Bala I \& Venkat V. (2019). "Competing in digital ecosystems," Business Horizons, Elsevier, vol. 62(1), pages 83-94.

[3] Kapoor, R. (2018) Ecosystems: broadening the locus of value creation. J Org Design 7, 12 https://doi.org/10.1186/s41469-018-0035-4

[4] Annabelle G, and Cusumano M. (2013) "Industry Platforms and Ecosystem Innovation.” J Prod Innov Manag 31, no. 3: 417-433.

[5] Rashi G. "Marketing in an Information-Intensive Environment: Strategic Implications of Knowledge as an Asset.” Journal of Marketing, vol. 55, no. 4, 1991, pp. 1-19. JSTOR, www.jstor.org/stable/1251953. Accessed 8 Sept. 2021.

[6] Polly Y, (2018) "Effect of pillow temperature on sleep"

[7] Harmat L, Takács J, Bódizs R. (2008) Music improves sleep quality in students. J Adv Nurs.
May;62(3):327-35. doi: 10.1111/j.13652648.2008.04602. x. PMID: 18426457.

[8] Hui-Ling L, Marion G, (2005), Music improves sleep quality in older adults, https://doi.org/10.1111/j.1365-2648.2004.03281.x

[9] WangSun, Z Jul 13, 2021 (The Expresswire) -- In 2021, " Smart Pillows Market " Size, Status and Market Insights, Forecast to 2027 |( Number of Pages:117)

[10] Subramaniam, M. (2020) Digital ecosystems and their implications for competitive strategy. J Org Design 9, 12. https://doi.org/10.1186/s41469-02000073-0

[11] Kotler P., Armstrong. G, Principles of Marketing, (2018) , published by Pearson Education, United Kingdom 